\title{
Energy-efficient renewable scheme for rechargeable sensor networks
}

\author{
Qian Zhang ${ }^{*}$, Rong Cheng and Zhihua Zheng
}

\begin{abstract}
Wireless energy transfer (WET) is a promising technology to fundamentally settle energy and lifetime problems in a wireless sensor network (WSN). In this paper, we study the operation of WSN based on WET using a mobile charging vehicle (MCV) and construct a periodic strategy to make the network operational permanently. Our goal is to decrease energy consumption of the entire system while maintaining the network operational forever. Based on the analysis of total energy consumption, we propose an energy-efficient renewable scheme (ERSVC) to achieve energy saving. Compared to previous schemes where the MCV visits and charges all nodes in each cycle, the MCV only needs to visit a portion of nodes in ERSVC. Numerical results show that our scheme can significantly decrease the total energy consumption with no performance loss. It is also validated that ERSVC can maintain the network operational forever with lower complexity than other schemes, making it more practical for real networks.
\end{abstract}

Keywords: Wireless energy transfer, Energy-efficient, Renewable, Wireless sensor network

\section{Introduction}

Wireless sensor networks (WSNs) nowadays are primarily powered by batteries. Due to limited energy capacity in a battery at each node, a WSN can only remain operational for limited time. To prolong its lifetime, there have been extensive researches in the last decade [1-4]. However, lifetime remains a performance bottleneck of a WSN and is one of the main reasons that limit its widespread application.

Recently, wireless energy transfer (WET) based on magnetic resonant coupling [5] was shown to be a promising technology to solve energy and lifetime problems in WSNs fundamentally [6-9]. Surprisingly, this new technology is immune to the surrounding environment. Compared with other WET technologies such as electro-magnetic radiation [10,11], magnetic resonant coupling has many significant advantages including higher energy conversion efficiency, further transmission distance, and no requirement of line-of-sight.

*Correspondence: zhangq@szpt.edu.cn

'Department of Electrical and Communication Engineering, Shenzhen

Polytechnic, Shahexi Road, 518055 Shenzhen, China
Some researchers investigated the problems in WSNs based on this non-radiative energy transfer. In [6], the authors proposed a model to make the WSN operational forever, with the objective of maximizing the ratio of vacation time. In [7], the authors extended the research in [6] to a dense network where several sensors could be charged at the same time. The authors in $[6,7]$ assumed that the mobile charger must visit all of the sensors in each cycle. However, sensors may have different energy consumption rate in real networks. Charging all nodes in each cycle will increase the traveling distance of MCV and thus degrading the energy efficiency of the entire system. In $[8,9]$, the authors studied the approach of combining mobile recharging and data gathering to further reduce energy consumption. These schemes assumed that the transmitter was omni-directional and could charge several nodes at the same time. However, the assumed scenarios in $[8$, 9] are difficult to be deployed in practice. Since magnetic resonant coupling has strict requirement with distance and orientation, which will make an effect on the charging efficiency [12]. Another drawback is that the complexities in $[8,9]$ are very high, causing them not suitable to be implemented in real networks.
Springer Open

(c) The Author(s). 2020 Open Access This article is licensed under a Creative Commons Attribution 4.0 International License, which permits use, sharing, adaptation, distribution and reproduction in any medium or format, as long as you give appropriate credit to the original author(s) and the source, provide a link to the Creative Commons licence, and indicate if changes were made. The images or other third party material in this article are included in the article's Creative Commons licence, unless indicated otherwise in a credit line to the material. If material is not included in the article's Creative Commons licence and your intended use is not permitted by statutory regulation or exceeds the permitted use, you will need to obtain permission directly from the copyright holder. To view a copy of this licence, visit http://creativecommons.org/licenses/by/4.0/. 
In this paper, we propose an energy-efficient renewable scheme (ERSVC) with low complexity based on this nonradiative energy transfer. Our goal is to decrease energy consumption of the entire system while maintaining the network operational permanently. Due to the potential large coverage of a WSN, we employ a mobile charging vehicle $(\mathrm{MCV})$ to periodically visit each sensor node and charge it wirelessly. Different from previous schemes in [6-8] , the MCV only visits and charges a portion of sensor nodes in ERSVC during each cycle. The set of charged nodes during each cycle is redesigned by taking into account the power consumption of each node. We use the total power consumption and MCV's vacation time ratio as the performance metric. Morevover, we derive the proof theoretically that ERSVC can make sure the network operational forever. Numerical results have shown that ERSVC achieves the goal of energy saving with lower complexity than other schemes in [6-9], which suggest it seems to be more practical for real applications.

The main contributions of this paper include several aspects. Firstly, we study the operation of a sensor network and construct a periodic strategy to make the network operational permanently. Secondly, we analyze the total energy consumption and point out that the traveling distance of $\mathrm{MCV}$ is the main factor influencing total energy consumption. Therefore, we primarily aim to decrease the traveling distance of MCV as much as possible in proposed ERSVC. Thirdly, we develop a comprehensive design for data flow, charging period and visiting set in ERSVC to achieve energy saving. Furthermore, we also demonstrate that ERSVC can make the network immortal theoretically.

\section{Methods}

In this section, we present the model for MCV's behavior and the control strategy in WSN. Table 1 lists the main abbreviations and notations in this paper.

\subsection{MCV and travel path}

We consider a sensor network $\mathcal{N}$ deployed over a twodimensional area, similar to the scenario that was widely adopted in [6-8]. Each sensor node has a battery capacity of $E_{\max }$ and is fully charged initially. Also, denote $E_{\min }$ as the minimum energy at a sensor node battery (for it to be operational). Each sensor node $i$ generates sensing data with a rate of $R_{i}$ (in bit/s), $i \in \mathcal{N}$. Inside the network, there is a fixed base station $(B)$, which is the sink node for all data generated by the sensor nodes. Multi-hop data routing can be employed for forwarding data by the sensor nodes.

To charge the battery at each sensor node, a mobile wireless charging vehicle (MCV) is employed in the network. As shown in Fig. 1, the MCV starts from a service station (denoted as origin $O$ ), and the traveling speed of
Table 1 Abbreviations and notations

\begin{tabular}{|c|c|}
\hline WSN & Wireless sensor network \\
\hline WET & Wireless energy transfer \\
\hline ERSVC & $\begin{array}{l}\text { Energy-efficient renewable scheme with variable cycle } \\
\text { (proposed scheme) }\end{array}$ \\
\hline TSP & Traveling salesman problem \\
\hline ECPM & Energy consumption per mile \\
\hline MCV & Mobile charging vehicle \\
\hline$E_{\min }$ & Minimum energy required to keep a sensor node operational \\
\hline $\mathcal{N}$ & The set of sensor nodes in the network \\
\hline O & The service station \\
\hline B & The base station \\
\hline$E_{\max }$ & Full battery capacity at a sensor node \\
\hline$R_{i}$ & Data rate generated by node $i$ \\
\hline$p_{i}$ & The power consumption of node $i$ \\
\hline$t_{i}$ & Charging time of node $i$ \\
\hline V & Traveling speed of MCV \\
\hline$U$ & Energy transfer rate of MCV \\
\hline$T$ & Period of a trip cycle of MCV \\
\hline$T_{i}$ & Charging period of node $i$ \\
\hline$T_{i}^{\prime}$ & Time interval between two adjacent arrivals at node $i$ \\
\hline$x_{i j}\left(\right.$ or $\left.x_{i B}\right)$ & Flow rate from node $i$ to node $j$ (or base station) \\
\hline$\beta_{i j}\left(\operatorname{or} \beta_{i B}\right)$ & $\begin{array}{l}\text { Power consumption for transmitting a unit of data from node } \\
\text { to node } j \text { (or base station) }\end{array}$ \\
\hline$\beta_{r}$ & Power consumption for receiving a unit of data rate \\
\hline$d_{i j}$ & Distance between node $i$ and node $j$ \\
\hline$\alpha$ & Path loss index \\
\hline$P_{\text {total }}$ & Total power consumption in the system \\
\hline$\lambda$ & Efficiency of non-radiative energy transfer \\
\hline$\eta_{\text {vac }}$ & The ratio of MCV's vacation time \\
\hline$t_{\text {vac }}$ & MCV's vacation time at service station \\
\hline r & The number of set needs to be classified \\
\hline$S_{k}$ & The defined set needs to be classified \\
\hline$F_{j}$ & The set of nodes which should be visited during the jth cycle \\
\hline$P_{j}$ & The traveling path of MCV during the jth cycle \\
\hline
\end{tabular}

the MCV is $V$ (in $\mathrm{m} / \mathrm{s}$ ). When it arrives at node $i$, it will spend a time of $t_{i}$ to charge the node's battery wirelessly via non-radiative energy transfer. Denote $U$ as the energy transfer rate of the MCV. After $t_{i}$, the MCV leaves node $i$ and travels to the next node.

After the MCV visits, all the sensor nodes that need to be charged in this cycle will return to the service station to be serviced (e.g., replacing or recharging its battery) and get ready for the next trip. We call this resting period vacation time, denoted as $t_{\mathrm{vac}}$.

For a given set of nodes $S$, the MCV starts from $O(O \in$ $S)$, visits and charges all nodes $n_{i}\left(n_{i} \in S\right)$, and finally 


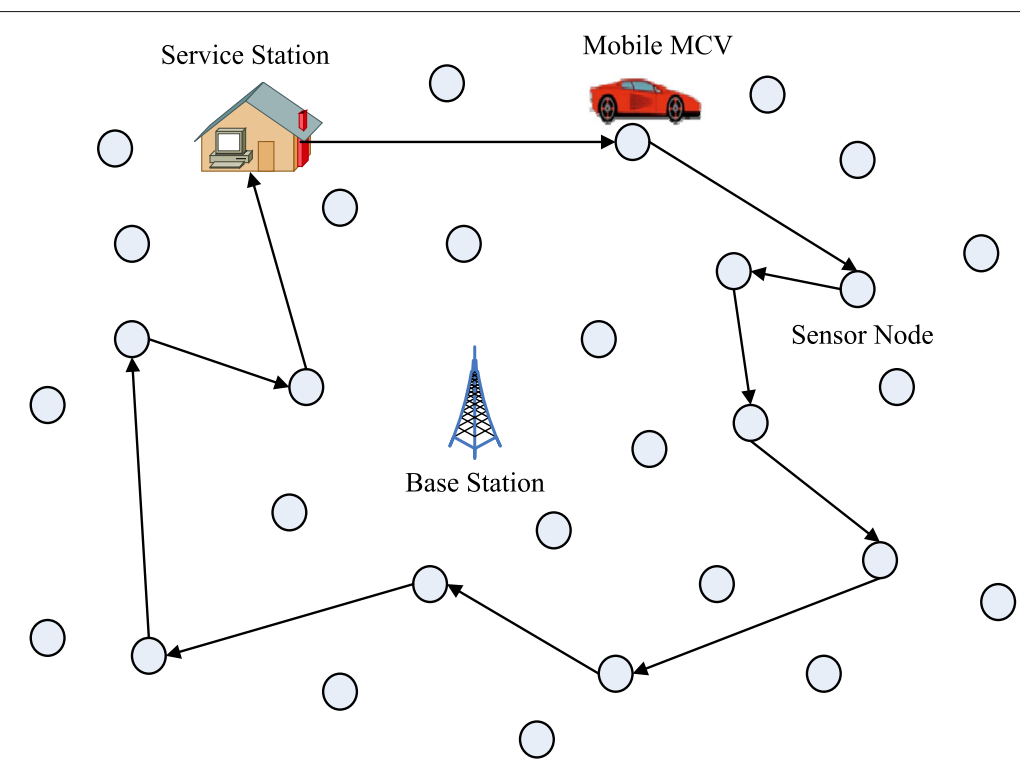

Fig. 1 A wireless sensor network with a mobile charging vehicle (MCV). This is the model for MCV's behavior. During each cycle, the MCV starts from a service station, visits and charges some nodes in the network, and finally returns to the service station

returns to $O$. This problem is defined as the traveling salesman problem (TSP) [13]. In TSP, the shortest path which connects all the nodes in $S$ and has the shortest overall length is called the shortest Hamiltonian cycle. It was proved in [6] that the MCV must move along the shortest Hamiltonian cycle in an optimal solution. It is obvious that the shortest Hamiltonian cycle is also the best path to achieve the energy-efficient optimization.

In each cycle, the MCV needs to visit and charge some nodes in the network. Denote $F_{k}$ as the set of nodes which need to be visited in the $k$ th cycle. In the $k$ th cycle, the MCV travels along the shortest Hamiltonian cycle which connects all nodes in $F_{k}$ and $O$. Denote $P_{k}$ as the traveling path of this shortest Hamiltonian cycle. Denote $D_{k}$ as the distance of path $P_{k}$, and $t_{P}=D_{k} / V$ as the time spent for traveling over distance $D_{k}$.

Denote $T$ as the period for a trip cycle of MCV, and $t_{\mathrm{vac}}^{k}$ as the vacation time of MCV in the $k$ th cycle. In the $k$ th cycle, the MCV travels from $O$, visits and charges all nodes in $F_{k}$, and finally returns to $O$ for a vacation time $t_{\mathrm{vac}}^{k}$. Then, the cycle time $T$ can be written as

$$
T=t_{P}+t_{\mathrm{vac}}^{k}+\sum_{j \in F_{k}} t_{j}=D_{k} / V+t_{\mathrm{vac}}^{k}+\sum_{j \in F_{k}} t_{j}
$$

where $\sum_{j \in F_{k}} t_{j}$ is the total amount of time that $\mathrm{MCV}$ spends charging all nodes in $F_{k}$ via non-radiative energy transfer.

\subsection{Control strategy}

In this paper, we use periodic strategy to make sure none of the sensor nodes runs out of energy. As mentioned above, a MCV is employed to charge the sensor nodes periodically with a cycle time of $T$. On the other hand, each node should be charged in time to supplement its energy consumption.

In previous researches [6-9], the MCV visits and charges all the nodes in each cycle. However, sensors may have different energy consumption rate in real networks. Some nodes which are close to the base station may consume energy several times higher than the remote ones and it is unnecessary to visit all nodes in each cycle. Therefore, we can adopt some strategies by taking into account the energy consumption rate of each node. For example, the node with the highest energy consumption rate will be visited in each cycle. In contrast, the node with lower energy consumption can be visited every 2 cycles or more.

For each node $i(i \in \mathcal{N})$, it should be recharged periodically to supplement its energy consumption in time. Denote $T_{i}$ as the charging period of node $i$. The charging process for node $i$ is shown in Fig. 2.

Denote $p_{i}$ as the energy consumption rate of node $i$. According to the energy conservation principle, the energy consumption of node $i$ should be equal to the energy supplied by MCV. Therefore, we have the following relationship,

$$
T_{i} \cdot p_{i}=t_{i} \cdot U(i \in \mathcal{N})
$$

\section{Analysis on total energy consumption}

In this section, we analyze the energy consumption of the entire system with MCV. For convenience of comparison, we use a plug-in hybrid vehicle (i.e., PHEV) to carry the mobile battery as the MCV. The total energy consumption consists of two parts as follows: 


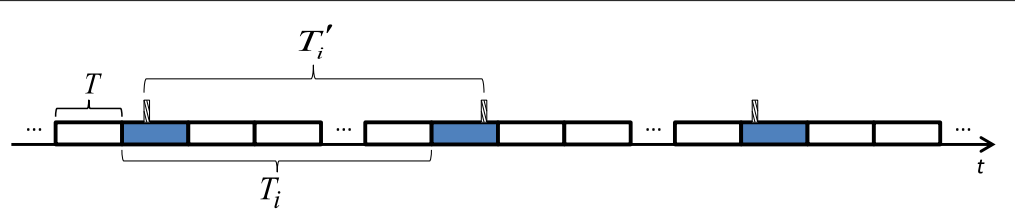

\begin{tabular}{|l}
$\mathbb{1} \quad$ WCV is charging the node $i$ \\
$\square \quad$ node $i$ will be charged in this cycle \\
$\square \quad$ node $i$ will not be charged in this cycle
\end{tabular}

Fig. 2 The charging period of node $i$ in WSN. For each node $i$, the MCV charges $i$ periodically with a mean period time of $T_{i}$. $T_{i}$ is set to be an integral multiple of $T$. The blue and white blocks indicate that $i$ will be charged and not be charged in this cycle, respectively. $T_{i}{ }^{\prime}$ is the visiting and charging interval of $i$, which is defined and explained detailly in the discussion section

- The energy that the $\mathrm{MCV}$ needs to charge all the sensor nodes in the network.

- The energy consumed by the MCV to travel inside the network.

According to the research data given by the Pacific Northwest National Laboratory [14], the energy consumption per mile (i.e., ECPM) of a mid-size PHEV is $0.3 \mathrm{~kW}$, that is, $675 \mathrm{~J} / \mathrm{m}$. Suppose the traveling speed of the $\mathrm{MCV}$ is $V=5 \mathrm{~m} / \mathrm{s}$, then the power consumed by the MCV traveling is about $3 \mathrm{~kW}$. However, the power consumption of a sensor node is about several milliwatts to several hundreds of milliwatts [1]. Apparently, the power consumed by the MCV traveling is the main part of the total energy consumption in the system.

In this paper, we propose an energy-efficient renewable scheme with variable cycle (ERSVC). In ERSVC, we firstly set the value of $T$ properly, then we design the charging period $T_{i}$ for each node $i$ based on its energy consumption rate $p_{i}$. The value of $T_{i}$ is set to be an integral multiple of $T$. Thus, the MCV can visit a portion of nodes in each cycle, and the traveling distance of MCV can be decreased. We use two major steps to decrease the two parts of total energy consumption respectively. We primarily aim to decrease the traveling distance of $\mathrm{MCV}$ as much as possible, since it is the main part of total energy consumption.

We use two parameters to measure the performance of network, which are stated as follows:

- The total power consumption (denoted as $P_{\text {total }}$ ), which is the sum of the two parts mentioned above. Denote $\lambda$ as the energy conversion efficiency of non-radiative energy transfer. Then, $P_{\text {total }}$ can be written as follows:

$$
P_{\text {total }}=\frac{1}{\lambda} \cdot \sum_{i, i \in \mathcal{N}} p_{i}+\frac{L_{\text {total }} \cdot \text { ECPM }}{T_{\text {total }}}
$$

where $T_{\text {total }}$ and $L_{\text {total }}$ are the total time and traveling distance of MCV over all of the cycles, respectively, and ECPM is defined as the energy consumption per mile in [14].

Note that all the sensor nodes in WSN are powered by electric energy converted from WET. Therefore, the first part of total power consumption can be written as $\frac{1}{\lambda} \cdot \sum_{i, i \in \mathcal{N}} p_{i}$.

- The ratio of the MCV's vacation time (denoted as $\eta_{\text {vac }}$ ), which is the optimization objective in [6-9]. In this paper, we define $\eta_{\mathrm{vac}}$ as the mean percentage of time in each cycle that MCV spends on its vacation, and it can be calculated by the following equation:

$$
\eta_{\mathrm{vac}}=\frac{\sum_{k} t_{\mathrm{vac}}^{k}}{T_{\mathrm{total}}}, \quad \eta_{\mathrm{vac}} \in[0,1]
$$

where $\sum_{k} t_{\mathrm{vac}}^{k}$ is the total amount of time that MCV spends on its vacation over all of the cycles.

In this paper, our goal is to decrease the value of $P_{\text {total }}$ while maintaining the network operational forever. In (4), we can see that when $\eta_{\mathrm{vac}}$ increases, it means that $\mathrm{MCV}$ has more time to replace or recharge its battery at the service station, which indicates better performance of the network.

\section{Implementation of proposed ERSVC}

In this section, we present the procedure of ERSVC. The implementation of ERSVC includes two major steps. The first step is to optimize the first part of total energy consumption (i.e., $\sum_{i, i \in \mathcal{N}} p_{i}$ ). In the second step, we make a joint design to decrease the traveling distance of MCV.

\subsection{Optimization with flowing rate and data routing}

Denote $x_{i j}$ as the flow rate from node $i$ to node $j$ and $x_{i B}$ as the flow rate from node $i$ to the base station $B$, respectively. Then, we have the following flow balance constraint [6] at each node $i$,

$$
\sum_{k \in \mathcal{N}}^{k \neq i} x_{k i}+R_{i}=\sum_{j \in \mathcal{N}}^{j \neq i} x_{i j}+x_{i B} \quad(i \in \mathcal{N})
$$


Each sensor node consumes energy for data transmission and reception. In this paper, we use the following energy consumption model [1] which is widely used in previous researches [6-8].

$$
p_{i}=\beta_{r} \cdot \sum_{k \in \mathcal{N}}^{k \neq i} x_{k i}+\sum_{j \in \mathcal{N}}^{j \neq i} \beta_{i j} \cdot x_{i j}+\beta_{i B} \cdot x_{i B} \quad(i \in \mathcal{N})
$$

where $\beta_{r}$ is the energy consumption rate for receiving a unit of data rate, $\beta_{i j}$ (or $\beta_{i B}$ ) is the energy consumption rate for transmitting a unit of data rate from node $i$ to node $j$ (or the base station $B$ ). Furthermore, $\beta_{i j}=C_{1}+C_{2} \cdot d_{i j}^{\alpha}$, where $d_{i j}$ is the distance between node $i$ and node $j, C_{1}$ is a distance independent constant term, $C_{2}$ is a coefficient of the distance dependent term, and $\alpha$ is the path loss index. In this model, $\beta_{r} \cdot \sum_{k \in \mathcal{N}}^{k \neq i} x_{k i}$ is the energy consumption rate for reception, and $\sum_{j \in \mathcal{N}}^{j \neq i} \beta_{i j} \cdot x_{i j}+\beta_{i B} \cdot x_{i B}$ is the energy consumption rate for transmission.

We assume that the flow rate $\left(x_{i j}\right.$ and $\left.x_{i B}\right)$ in the network is invariant with time. The optimization objective is the total power consumption of all sensor nodes (i.e., $\sum_{i, i \in \mathcal{N}} p_{i}$ ) which is the first part of $P_{\text {total }}$ in (3). Each node should satisfy the basic flow balance constraint in (5) and energy consumption model in (6). Therefore, the optimal problem can be formulated to a linear programming problem as follows:

$$
\begin{array}{ll}
\min & \sum_{i, i \in \mathcal{N}} p_{i} \\
\text { s.t. } & \sum_{k \in \mathcal{N}}^{k \neq i} x_{k i}+R_{i}=\sum_{j \in \mathcal{N}}^{j \neq i} x_{i j}+x_{i B} \\
& p_{i}=\beta_{r} \cdot \sum_{k \in \mathcal{N}}^{k \neq i} x_{k i}+\sum_{j \in \mathcal{N}}^{j \neq i} \beta_{i j} \cdot x_{i j}+\beta_{i B} \cdot x_{i B} \\
& 0 \leq p_{i} \leq \kappa \\
& x_{i j} \geq 0
\end{array}
$$

In this problem, $x_{i j}, x_{i B}$ and $p_{i}$ are the optimization variables. $R_{i}, \beta_{r}, \beta_{i j}$, and $\beta_{i B}$ are constants. $\kappa$ is a constant which is defined in the following Theorem 1 .

Define $D_{T S P}$ as the distance of the shortest Hamilton cycle which connects all nodes in $\mathcal{N}$ and the service station $O$. Then, the system should satisfy a feasibility condition which is stated in Theorem 1 as follows.

Theorem 1 For any node $i \quad(i \in \mathcal{N})$, the optimization problem has a feasible solution when $p_{i}$ satisfies the following condition.

$$
0 \leq p_{i} \leq \frac{A U+(2 N+1) E_{1}-\sqrt{A^{2} U^{2}+(2 N+1)^{2} E_{1}^{2}+2(2 N-1) A U E_{1}}}{2 A}
$$

where $N$ is the number of sensor nodes in the network, $A=2 D_{T S P} / V$ and $E_{1}=E_{\max }-E_{\min }$. The right value in (8) is a constant, which is defined as $\kappa$. The proof of Theorem 1 is given in the "Discussion" section.

Note that the constrained condition in Theorem 1 is set up to ensure that the vacation time meets the condition $t_{\mathrm{vac}}^{k} \geq 0$ in (1), which means the sum of the traveling time of $\mathrm{MCV}$ and the charging time for all nodes should not be greater than the total cycle time $T$.

\subsection{Procedure of joint design}

We can obtain the power consumption $p_{i}$ of each node $i$ after solving the optimization problem in (7). In this subsection, we design the system for combining the charging period $T_{i}$, the visiting set $F_{k}$ in each cycle, and the traveling path of MCV. The steps are as follows.

\subsubsection{Step 1}

In step 1 , we set the value of $T$ for MCV and the number of set needs to be classified.

Firstly, compute the maximum and minimum of $p_{i}$ respectively as follows:

$$
p_{\max }=\max p_{i}, \quad p_{\min }=\min p_{i}
$$

Secondly, set the value of $T$, and the maximum charging period (denoted as $T_{\max }$ ) corresponding to the node which has the minimum power consumption as follows:

$$
T=\frac{E_{\max }-E_{\min }}{2 \cdot p_{\max }}, \quad T_{\max }=\frac{E_{\max }-E_{\min }}{p_{\min }}
$$

Denote $r$ as the number of set that needs to be classified, which is set up as follows:

$$
r=\left\lceil\log _{2}\left\lfloor T_{\max } / T\right\rfloor\right\rceil
$$

where \lceil\rceil and $L\rfloor$ is the operating of making a number round up to and down to the nearest integer, respectively.

\subsubsection{Step 2}

In this step, we set the charging period $T_{i}$ for each node $i$ and classify the set $S_{k}$.

Firstly, for each node $i(i \in \mathcal{N})$, we set its charging period $T_{i}$ as follows:

$$
T_{i}=2^{a-1} \cdot T \quad(1 \leq a \leq r)
$$

where $a$ is the approximate logarithm of the ratio of $T_{i}$ and $T$ and is calculated as follows:

$$
a=\left\lfloor\log _{2}\left(\frac{E_{\max }-E_{\min }}{p_{i} \cdot T}-1\right)\right\rfloor+1
$$

Define set $S_{k}(1 \leq k \leq r)$ and let $i \in S_{a}$, then the MCV should visit node $i$ in the $\left(n \cdot 2^{a-1}\right)$ th trip cycle. Note that we multiply the parameter $T$ by coefficient $1 / 2$ in (10) to guarantee the paramenter $a$ meets the condition $a \geq 1$. 


\subsubsection{Step 3}

In this step, we will obtain the visiting set $F_{j}$ of MCV during the $j$ th cycle and design the traveling path of MCV. We can express $j\left(1 \leq j \leq 2^{r-1}\right)$ as $j=m \cdot 2^{c}$, where $m$ is an odd number, $c$ is an integer, and $c \geq 0$. During the $j$ th cycle, denote $F_{j}$ as the set of nodes which should be visited and recharged, then $F_{j}$ can be obtained as follows:

$$
F_{j}= \begin{cases}S_{1} & (c=0) \\ S_{1} \cup S_{2} \ldots \cup S_{c+1} & (c \geq 1)\end{cases}
$$

Denote $P_{j}$ as the traveling path of MCV during the $j$ th cycle. It is obvious that $P_{j}$ should be the shortest Hamilton cycle which connects all nodes in $F_{j}$ and the service station $O$, that is,

$$
P_{j}=\operatorname{Hamiltonian}\left(F_{j} \cup O\right)
$$

During the $j$ th cycle, for each node $n_{i} \in F_{j}$, the MCV travels to $n_{i}$ and charges its battery to $E_{\max }$. After $2^{r-1}$ cycles, the MCV will recount from the first cycle and keep circulating like this.

The detailed steps of this subsection are summarized in Algorithm 1.

\section{Numerical results}

In this section, we present some numerical results to explain how ERSVC works in a real network. In order to evaluate the performance for proposed ERSVC and other schemes in previous researches, we use the network topology and parameter settings similar to those in [6-9]. The simulations are conducted with MatLab software.

\subsection{Simulation settings}

We consider two randomly generated WSNs consisting of 50 and 100 nodes, respectively. The sensor nodes are deployed randomly in a square area of $1 \times 1 \mathrm{~km}^{2}$. Both the base station and the service station are assumed to be located at $(500,500)$ (in $\mathrm{m}$ ). The data rate $R_{i}$ from each node $i$ is randomly generated within $[1,10] \mathrm{kb} / \mathrm{s}$. The parameters in (6) are $C_{1}=50 \mathrm{~nJ} / \mathrm{b}, C_{2}=0.0013 \mathrm{pJ} /(\mathrm{b}$. $\left.\mathrm{m}^{4}\right), \beta_{r}=50 \mathrm{~nJ} / \mathrm{b}$, and $\alpha=4$ [6-8]. The traveling speed of $\mathrm{MCV}$ is $V=5 \mathrm{~m} / \mathrm{s}$, and the energy transfer rate of $\mathrm{MCV}$ is $U=5 \mathrm{~W}$ which is well within feasible range [5]. We assume the charging distance between MCV and each sensor node is about $1 \mathrm{~m}$. Thus, the value of energy conversion efficiency can be set to $\lambda=0.85$ [5].

For a sensor node's battery, we choose a regular NiMH battery and its cell voltage and the quantity of electricity is $1.2 \mathrm{~V} / 2.5 \mathrm{Ah}$. Let $E_{\max }=10.8 \mathrm{KJ}$, and $E_{\min }=0.05 \cdot E_{\max }=$ $540 \mathrm{~J}[6]$.

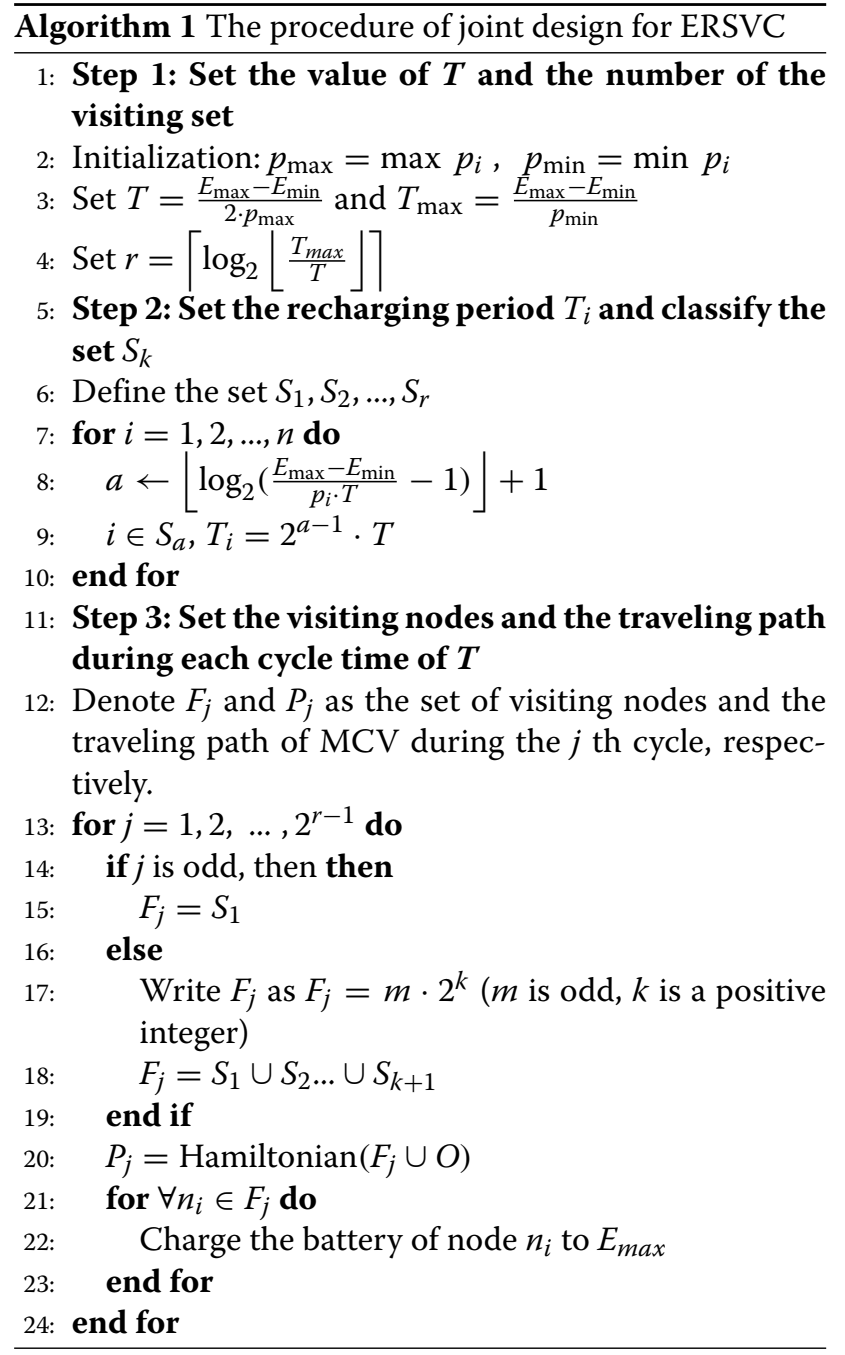

\subsection{Results}

\subsubsection{0-Node network}

We firstly present the results for the 50-node network. Table 2 gives the location of each node and its data rate for a 50-node network.

Figure 3 illustrates the data routing result after optimizing in the first step of ERSVC. In Fig. 3, an arrow from node $i$ to node $j$ indicates that there is data transmitting from node $i$ to node $j$ (i.e., $x_{i j}>0$ ).

After the first step, we can obtain the power consumption $p_{i}$ of node $i$ by (6). Then, the node with the highest power and the node with the lowest power can be calculated, which correspond to the 48th node and the 12th node, respectively. So the number of set needs to be classified is $r=12$, which can be calculated by (11). The result of the classified set $S_{k}$ is shown in Table 3.

In ERSVC, the period of a trip cycle is $T=14.4 \mathrm{~h}$. The optimal traveling path of MCV during the $\left(2^{5}=32\right)$ th cycle is shown in Fig. 4. According to (14), the visiting set of MCV is $F_{32}=S_{1} \cup S_{2} \cup \ldots \cup S_{6}$ in the 32th cycle. It 
Table 2 Location and data rate for each node in a 50-node network

\begin{tabular}{|c|c|c|c|c|c|c|c|c|}
\hline $\begin{array}{l}\text { Node } \\
\text { Index }\end{array}$ & $\begin{array}{l}\text { Location } \\
(\mathrm{m})\end{array}$ & $\begin{array}{l}R_{i} \\
(\mathrm{~kb} / \mathrm{s})\end{array}$ & $\begin{array}{l}\text { Node } \\
\text { Index }\end{array}$ & $\begin{array}{l}\text { Location } \\
\text { (m) }\end{array}$ & $\begin{array}{l}R_{i} \\
(\mathrm{~kb} / \mathrm{s})\end{array}$ & $\begin{array}{l}\text { Node } \\
\text { Index }\end{array}$ & $\begin{array}{l}\text { Location } \\
\text { (m) }\end{array}$ & $\begin{array}{l}R_{i} \\
(\mathrm{~kb} / \mathrm{s})\end{array}$ \\
\hline 1 & $(815,276)$ & 1 & 18 & $(916,139)$ & 10 & 35 & $(97,917)$ & 2 \\
\hline 2 & $(906,680)$ & 8 & 19 & $(792,149)$ & 1 & 36 & $(823,286)$ & 2 \\
\hline 3 & $(127,655)$ & 4 & 20 & $(959,258)$ & 5 & 37 & $(695,757)$ & 8 \\
\hline 4 & $(913,163)$ & 6 & 21 & $(656,841)$ & 3 & 38 & $(317,754)$ & 6 \\
\hline 5 & $(632,119)$ & 3 & 22 & $(36,254)$ & 10 & 39 & $(950,380)$ & 6 \\
\hline 6 & $(98,498)$ & 7 & 23 & $(849,814)$ & 1 & 40 & $(34,568)$ & 2 \\
\hline 7 & $(278,960)$ & 3 & 24 & $(934,244)$ & 8 & 41 & $(439,76)$ & 8 \\
\hline 8 & $(547,340)$ & 7 & 25 & $(679,929)$ & 8 & 42 & $(382,54)$ & 7 \\
\hline 9 & $(958,585)$ & 6 & 26 & $(758,350)$ & 9 & 43 & $(766,531)$ & 4 \\
\hline 10 & $(965,224)$ & 8 & 27 & $(743,197)$ & 1 & 44 & $(795,779)$ & 6 \\
\hline 11 & $(158,751)$ & 5 & 28 & $(392,251)$ & 4 & 45 & $(187,934)$ & 6 \\
\hline 12 & $(971,255)$ & 1 & 29 & $(655,616)$ & 4 & 46 & $(490,130)$ & 1 \\
\hline 13 & $(957,506)$ & 4 & 30 & $(171,473)$ & 9 & 47 & $(446,569)$ & 3 \\
\hline 14 & $(485,699)$ & 10 & 31 & $(706,352)$ & 5 & 48 & $(646,469)$ & 2 \\
\hline 15 & $(800,891)$ & 2 & 32 & $(32,831)$ & 10 & 49 & $(709,12)$ & 2 \\
\hline 16 & $(142,959)$ & 9 & 33 & $(277,585)$ & 1 & 50 & $(755,337)$ & 3 \\
\hline 17 & $(422,547)$ & 5 & 34 & $(46,550)$ & 3 & & & \\
\hline
\end{tabular}

is obvious that the optimal traveling path is the shortest Hamiltonian cycle. We can solve this problem by using the Concorde solver [15].

As shown in Fig. 4, different from other schemes in previous researches [6-8], the MCV only needs to charge a portion of nodes during the 32th cycle, which is consistent with our previous analysis. After $2^{r-1}=2048$ cycles, the $\mathrm{MCV}$ will recount from the first cycle and so on.

The energy cycle behavior of the 48th node which has the highest power consumption is shown in Fig. 5. We can see that the remaining energy of the battery at the 48th node is always higher than $E_{\text {min }}$ during a charging period. Thus, it will remain operational forever due to the periodicity of each node in the network.

After the implementation of ERSVC, we can finally compute the total power consumption $P_{\text {total }}$ by (3). Then, the vacation time ratio $\eta_{\mathrm{vac}}$ can be obtained by bringing (2) into (1). $P_{\text {total }}$ and $\eta_{\mathrm{vac}}$ are two parameters to measure the performance of the network. In traditional scheme (such as $[6,7])$, the MCV visits and charges all the nodes in the network with constant cycle time, and we call it the traditional scheme with constant cycle (TSCC). Table 4 illustrates these two parameters for proposed ERSVC, TSCC, and the scheme in [9].

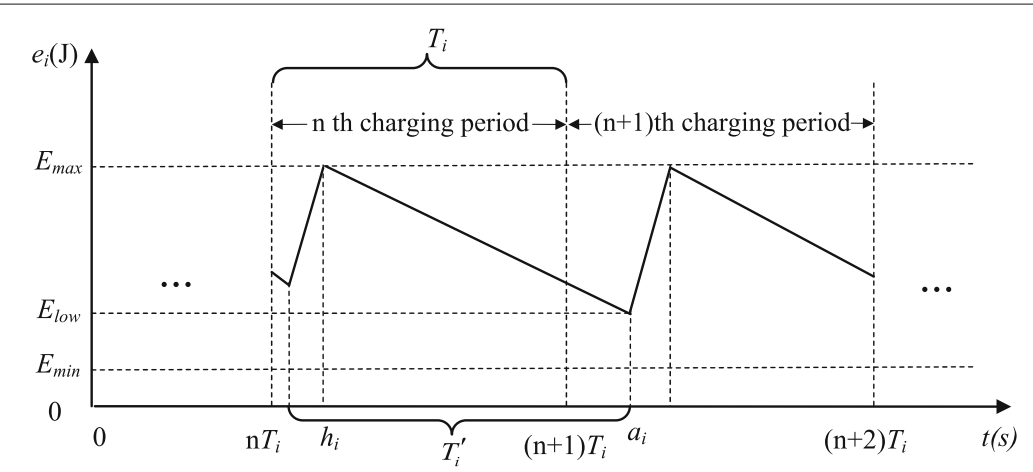

Fig. 3 Data routing for proposed ERSVC in the 50-node network. After implementing the first step of ERSVC, we can obtain the dating routing result. Multi-hop data routing is employed for forwarding data by the sensor nodes. In this figure, an arrow from node $i$ to node $j$ indicates that there is data transmitting from node $i$ to node $j$ 
Table 3 The result of the classified set $S_{k}(k=1,2, \ldots, r)$ for the 50-node network

\begin{tabular}{llll}
\hline Set Index & Node Index & \multicolumn{2}{l}{ Set Index } \\
\hline$S_{1}$ & 48 & $S_{7}$ & $1,4,6,20,21,41,43$ \\
$S_{2}$ & $3,22,31,33$ & $S_{8}$ & $25,45,49$ \\
$S_{3}$ & $8,29,30,32,37$ & $S_{9}$ & $7,9,10,15,16,18,26,42$ \\
$S_{4}$ & $17,24,28,46$ & $S_{10}$ & 34,40 \\
$S_{5}$ & $11,14,36,38,39,50$ & $S_{11}$ & none \\
$S_{6}$ & $2,5,13,27,35,44,47$ & $S_{12}$ & $12,19,23$ \\
\hline
\end{tabular}

In Table $4, \overline{L_{\text {total }}}(m)$ is defined as the average traveling distance of MCV over 2048 cycles. From Table 4, we can see that ERSVC decreases total energy consumption $P_{\text {total }}$ by about $48 \%$ compared to TSCC, while maintaining the vacation time ratio $\eta_{\mathrm{vac}}$ nearly equal to that in other schemes. In [9], the authors designed a cluster-based network topology and selected some nodes as cluster heads to reduce the traveling distance and total energy consumption. Nevertheless, the MCV still need to visit all nodes in each cycle in [9]. Therefore, ERSVC outperforms the scheme in [9] in terms of energy consumption.

In ERSVC, the total power consumption of all nodes is $\sum_{i, i \in \mathcal{N}} p_{i}=0.58$ and it is much less than $P_{\text {total }}$, as shown in Table 4. Therefore, we can deduce that the traveling distance of $\mathrm{MCV}$ (i.e., $\overline{L_{\text {total }}}$ ) is the main factor influencing $P_{\text {total }}$ in (3). In Table $4, \overline{L_{\text {total }}}$ is significantly decreased by about $75.4 \%$ compared to TSCC, since the MCV only needs to charge a portion of nodes in each cycle.

\subsubsection{0-Node network}

Table 5 gives the location of each node and its data rate for a 100-node network. Figure 6 illustrates the data routing result after optimizing in the first step of ERSVC.

The result of the classified set $S_{k}$ is shown in Table 6 . Table 7 shows the network performance measured by $P_{\text {total }}$ and $\eta_{\text {vac }}$ for proposed ERSVC and traditional TSCC.

In Table 7, we can see that ERSVC decreases the total energy consumption $P_{\text {total }}$ by about $51 \%$ and $42 \%$ compared to TSCC and the scheme in [9] respectively, with no performance loss in terms of the vacation time ratio $\eta_{\mathrm{vac}}$. Therefore, ERSVC outperforms TSCC and the scheme in [9] in terms of energy consumption and achieves the goal of energy saving.

\section{Discussion}

In this section, we firstly give the proof that ERSVC can make the network immortal. Then, we prove the Theorem 1 which is mentioned in previous implementation section.

\subsection{Proof of the network's sustainability with ERSVC}

In this subsection, we prove that ERSCV can guarantee the network operational permanently. Before giving the proof, we firstly prove a Lemma which is stated in Lemma 1.

Lemma 1 For any node $i(i \in \mathcal{N})$ in the network, the sufficient condition to make it immortal is that the visiting interval of $i$ satisfies inequation: $T_{i}^{\prime} \leq\left(E_{\max }-E_{\min }\right) / p_{i}$.

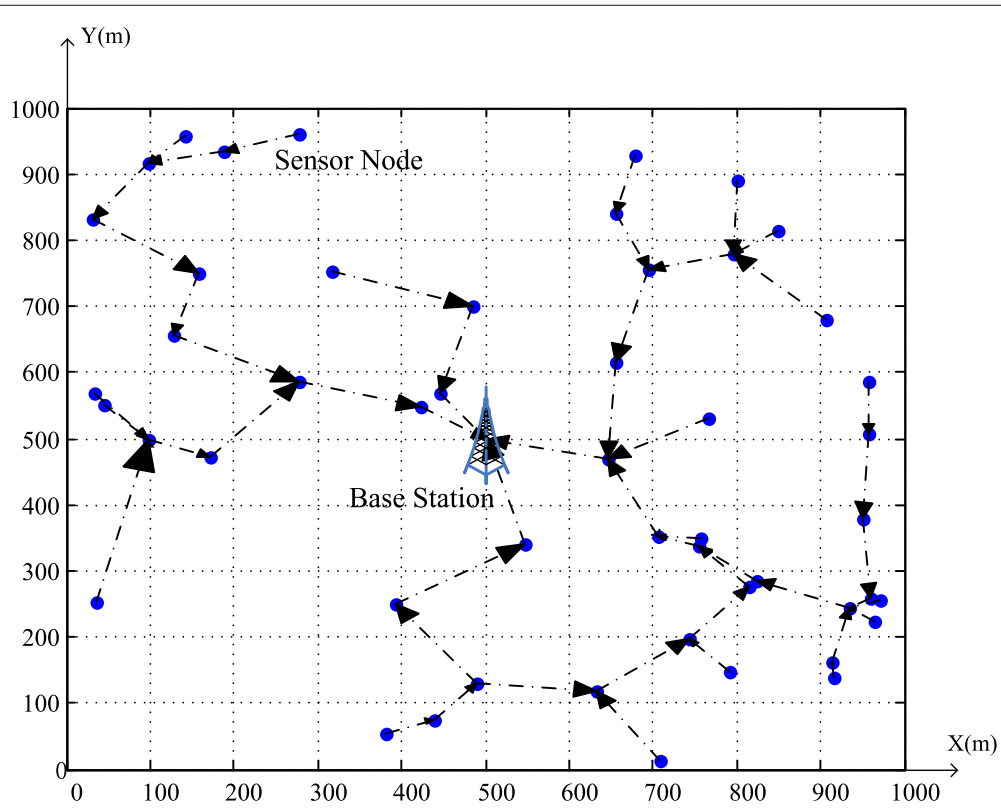

Fig. 4 The optimal traveling path of MCV during the 32th cycle. During the 32th cycle, the visiting set of $M C V$ is $F_{32}=S_{1} \cup S_{2} \cup \ldots \cup S_{6}$. Different from other schemes in previous researches, the MCV only needs to charge a portion of nodes during the 32th cycle. Based on previous analysis, the optimal traveling path is the shortest Hamiltonian cycle which connects all nodes in $F_{32}$ and the service station 


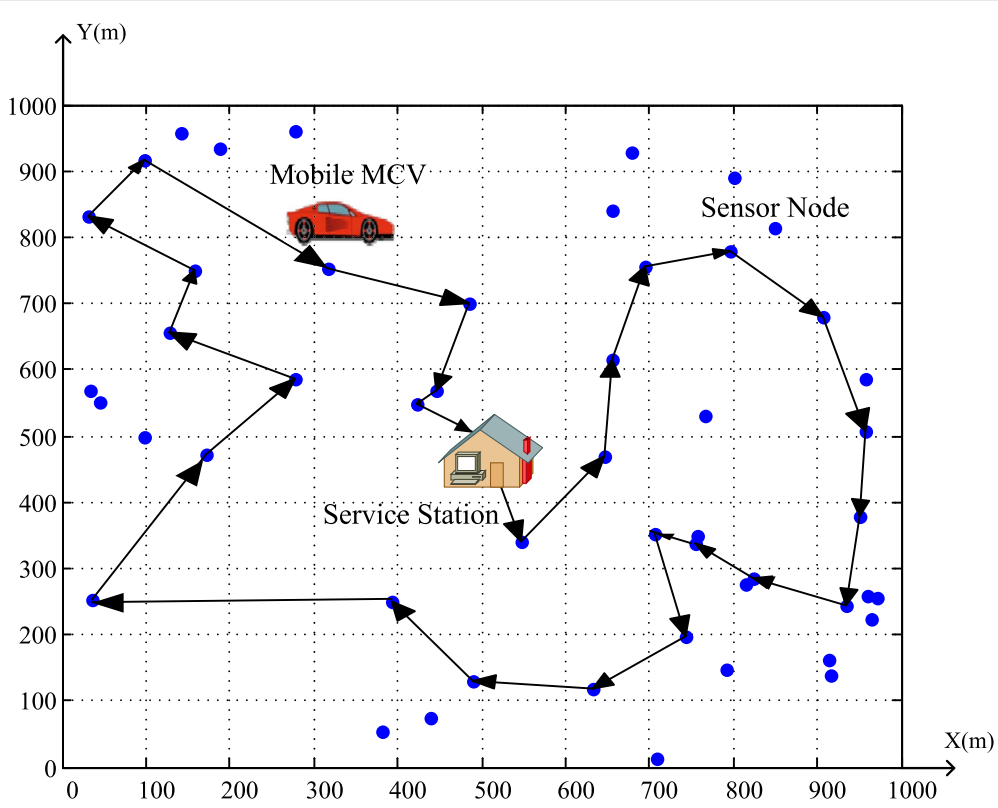

Fig. 5 The energy behavior of the 48th node during two charging periods. The 48th node has the highest power consumption among all nodes in the network. The energy behavior of each node is periodic and can be calculated after the implementation of ERSVC. Similarly, we can also obtain the energy behavior for other nodes. If the remaining energy of a node's battery is always higher than $E_{\min }$, it will remain operational forever

Proof Define $T_{i}^{\prime}$ the visiting and charging interval of $i$, which is the time interval between two adjacent arrivals at node $i$ by MCV. Figure 7 is the energy behavior of node $i$ during the $n$th and $(n+1)$ th charging period. Different from $T_{i}$ which is set to be an integral multiple of $T$, the value of $T_{i}{ }^{\prime}$ during each charging period is different and its mean value is $T_{i}$, as shown in Fig. 7 .

Denote $E_{\text {low }}$ as the lowest remaining energy of the battery at node $i$. To prove Lemma 1, we only need to prove that $E_{\text {low }}$ is greater than or equal to $E_{\mathrm{min}}$. As shown in Fig. 7, denote $a_{i}$ as the moment that MCV arrives at $i$ for the $(n+1)$ th time, and $h_{i}$ the moment when MCV charges the battery at node $i$ to $E_{\max }$ for the $n$th time. Assume the remaining energy of the battery at node $i$ is $E_{\text {low }}$ at $a_{i}$; therefore,

$$
\begin{aligned}
E_{\mathrm{low}}=E_{\max }-p_{i}\left(a_{i}-h_{i}\right) & \geq E_{\max }-p_{i} T_{i}^{\prime} \geq E_{\max } \\
& -p_{i}\left(E_{\max }-E_{\min }\right) / p_{i}=E_{\min }
\end{aligned}
$$

where the second inequation is based on the sufficient condition in Lemma 1. This completes the proof.

Table 4 Comparison of performance for proposed ERSVC and TSCC in the 50-node network

\begin{tabular}{llll}
\hline Parameter & Proposed ERSVC & Traditional TSCC & Scheme in [9] \\
\hline$\overline{L_{\text {total }}}(m)$ & 1392 & 5663 & 4602 \\
$P_{\text {total }}$ & 18.33 & 35.14 & 30.28 \\
$\eta_{\text {vac }}$ & $87.88 \%$ & $87.27 \%$ & $87.31 \%$ \\
\hline
\end{tabular}

Theorem 2 For any node $i(i \in \mathcal{N})$ in the network, the proposed scheme ERSVC can make it operational forever.

In ERSVC, the MCV only visits and recharges a portion of nodes in each cycle, and the visiting set in each cycle is different. Therefore, the moment when MCV arrives at $i$ is different every time, and the visiting interval $T_{i}{ }^{\prime}$ is variable, as shown in Figs. 2 and 7. According to (12), we have $T_{i}=2^{a-1} \cdot T$. As shown in Fig. 2, we can obtain the following relationship,

$$
\left(2^{a-1}-1\right) \cdot T<T_{i}^{\prime}<\left(2^{a-1}+1\right) \cdot T
$$

Therefore, we can obtain the following result based on (13),

$$
\begin{aligned}
T_{i}^{\prime} & <\left(2^{a-1}+1\right) \cdot T=\left(2^{\left\lfloor\log _{2}\left(\frac{E_{\max }-E_{\min }}{p_{i} \cdot T}-1\right)\right\rfloor}+1\right) \cdot T \\
& \leq\left(\frac{E_{\max }-E_{\min }}{p_{i} \cdot T}-1+1\right) \cdot T=\left(E_{\max }-E_{\min }\right) / p_{i}
\end{aligned}
$$

According to Lemma 1 , for any node $i(i \in \mathcal{N})$ in the network, it can operate with unlimited time. This completes the proof.

\subsection{Proof of theorem 1}

Recall that the constrained condition in Theorem 1 is set up to ensure that the vacation time meets the condition $t_{\mathrm{vac}}^{k} \geq 0$ in (1). Therefore, we have the following inequality during the $k$ th cycle:

$$
T \geq D_{k} / V+\sum_{j \in F_{k}} t_{j}
$$


Table 5 Location and data rate for each node in a 100-node network

\begin{tabular}{|c|c|c|c|c|c|c|c|c|}
\hline $\begin{array}{l}\text { Node } \\
\text { Index }\end{array}$ & $\begin{array}{l}\text { Location } \\
\text { (m) }\end{array}$ & $\begin{array}{l}R_{i} \\
(\mathrm{~kb} / \mathrm{s})\end{array}$ & $\begin{array}{l}\text { Node } \\
\text { Index }\end{array}$ & $\begin{array}{l}\text { Location } \\
\text { (m) }\end{array}$ & $\begin{array}{l}R_{i} \\
(\mathrm{~kb} / \mathrm{s})\end{array}$ & $\begin{array}{l}\text { Node } \\
\text { Index }\end{array}$ & $\begin{array}{l}\text { Location } \\
\text { (m) }\end{array}$ & $\begin{array}{l}R_{i} \\
(\mathrm{~kb} / \mathrm{s})\end{array}$ \\
\hline 1 & $(970,383)$ & 10 & 35 & $(921,680)$ & 4 & 68 & $(505,6)$ & 4 \\
\hline 2 & $(124,85)$ & 8 & 36 & $(498,557)$ & 3 & 69 & $(405,771)$ & 10 \\
\hline 3 & $(467,734)$ & 6 & 37 & $(278,851)$ & 2 & 70 & $(174,765)$ & 6 \\
\hline 4 & $(657,332)$ & 3 & 38 & $(653,559)$ & 4 & 71 & $(575,421)$ & 9 \\
\hline 5 & $(290,840)$ & 3 & 39 & $(917,902)$ & 7 & 72 & $(606,57)$ & 5 \\
\hline 6 & $(755,372)$ & 9 & 40 & $(510,420)$ & 3 & 73 & $(214,586)$ & 5 \\
\hline 7 & $(558,828)$ & 5 & 41 & $(974,358)$ & 7 & 74 & $(520,174)$ & 9 \\
\hline 8 & $(428,177)$ & 9 & 42 & $(197,489)$ & 5 & 75 & $(989,729)$ & 10 \\
\hline 9 & $(267,130)$ & 1 & 43 & $(111,256)$ & 5 & 76 & $(490,534)$ & 6 \\
\hline 10 & $(754,880)$ & 9 & 44 & $(297,929)$ & 2 & 77 & $(695,253)$ & 10 \\
\hline 11 & $(898,44)$ & 1 & 45 & $(396,467)$ & 2 & 78 & $(411,917)$ & 8 \\
\hline 12 & $(728,687)$ & 9 & 46 & $(421,254)$ & 7 & 79 & $(35,758)$ & 6 \\
\hline 13 & $(407,734)$ & 9 & 47 & $(311,431)$ & 3 & 80 & $(293,887)$ & 10 \\
\hline 14 & $(938,437)$ & 6 & 48 & $(694,703)$ & 6 & 81 & $(801,69)$ & 9 \\
\hline 15 & $(255,380)$ & 2 & 49 & $(92,402)$ & 10 & 82 & $(347,184)$ & 10 \\
\hline 16 & $(533,980)$ & 2 & 50 & $(402,182)$ & 8 & 83 & $(83,737)$ & 7 \\
\hline 17 & $(955,399)$ & 8 & 51 & $(295,856)$ & 7 & 84 & $(511,697)$ & 4 \\
\hline 18 & $(268,440)$ & 9 & 52 & $(306,584)$ & 10 & 85 & $(367,777)$ & 5 \\
\hline 19 & $(250,157)$ & 1 & 53 & $(106,374)$ & 8 & 86 & $(739,502)$ & 10 \\
\hline 20 & $(928,326)$ & 8 & 54 & $(594,222)$ & 7 & 87 & $(525,425)$ & 1 \\
\hline 21 & $(69,314)$ & 10 & 55 & $(283,219)$ & 10 & 88 & $(805,611)$ & 2 \\
\hline 22 & $(299,895)$ & 4 & 56 & $(155,522)$ & 1 & 89 & $(817,856)$ & 5 \\
\hline 23 & $(592,247)$ & 7 & 57 & $(1,433)$ & 3 & 90 & $(189,671)$ & 6 \\
\hline 24 & $(203,311)$ & 4 & 58 & $(284,741)$ & 10 & 91 & $(124,524)$ & 1 \\
\hline 25 & $(636,409)$ & 3 & 59 & $(551,70)$ & 8 & 92 & $(821,299)$ & 7 \\
\hline 26 & $(798,708)$ & 8 & 60 & $(871,847)$ & 5 & 93 & $(638,704)$ & 9 \\
\hline 27 & $(502,144)$ & 8 & 61 & $(42,680)$ & 7 & 94 & $(16,382)$ & 2 \\
\hline 28 & $(651,871)$ & 3 & 62 & $(905,137)$ & 5 & 95 & $(896,568)$ & 5 \\
\hline 29 & $(796,83)$ & 6 & 63 & $(131,858)$ & 4 & 96 & $(515,888)$ & 3 \\
\hline 30 & $(233,462)$ & 5 & 64 & $(834,200)$ & 3 & 97 & $(545,843)$ & 10 \\
\hline 31 & $(601,30)$ & 1 & 65 & $(800,607)$ & 4 & 98 & $(606,899)$ & 7 \\
\hline 32 & $(112,753)$ & 1 & 66 & $(918,543)$ & 1 & 99 & $(760,939)$ & 3 \\
\hline 33 & $(516,700)$ & 5 & 67 & $(137,162)$ & 5 & 100 & $(855,815)$ & 2 \\
\hline 34 & $(838,215)$ & 3 & & & & & & \\
\hline
\end{tabular}

Since $T$ is invarient in (19), we only need to prove that $T$ is not less than the maximum of the right value in (19). The maximum of the right value occurs when the visiting set $F_{k}$ includes all sensor nodes in the network (namely, $F_{k}=\mathcal{N}$ ), and we have $D_{k}=D_{T S P}$ at this time. Therefore, we have the following relationship,

$$
\frac{D_{k}}{V}+\sum_{j \in F_{k}} t_{j} \leq \frac{D_{T S P}}{V}+\sum_{j \in \mathcal{N}} t_{j}
$$

In Fig. 7, $a_{i}$ is the moment when MCV arrives at node $i$ in the $(n+1)$ th charging period. After the time of $t_{i}$, the MCV charges the battery of node $i$ to $E_{\max }$. Apparently, the slope is $\left(U-p_{i}\right)$ when the MCV is charging node $i$ at a rate of $U$. Therefore, we can obtain the following relationship:

$$
E_{\mathrm{max}}=E_{\mathrm{low}}+t_{i} \cdot\left(U-p_{i}\right) \geq E_{\mathrm{min}}+t_{i} \cdot\left(U-p_{i}\right)
$$




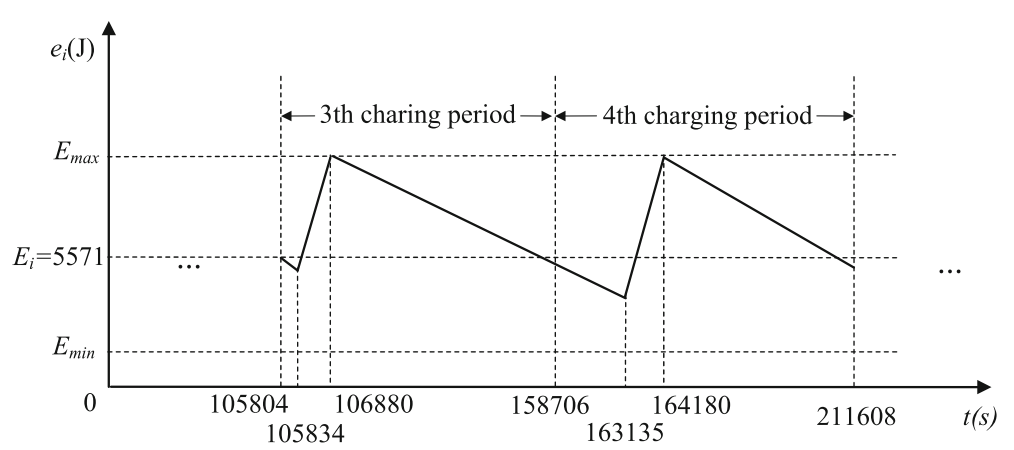

Fig. 6 Data routing for proposed ERSVC in the 100-node network. Similar to Fig. 3, this figure illustrates the data routing result for the 100-node network

Then, we can derive the following inequality based on (20) and (21):

$$
\begin{aligned}
\frac{D_{k}}{V}+\sum_{j \in F_{k}} t_{j} \leq \frac{D_{\mathrm{TSP}}}{V}+\sum_{j \in \mathcal{N}} t_{j} \leq & \frac{D_{\mathrm{TSP}}}{V}+\sum_{j \in \mathcal{N}} \frac{E_{1}}{U-p_{i}} \\
& \leq \frac{D_{\mathrm{TSP}}}{V}+\frac{N \cdot E_{1}}{U-p_{\max }}
\end{aligned}
$$

According to (10) , (19), and (22), the parameter $T$ should satisfy the following inequality to ensure the optimization problem in (7) has a feasible solution:

$$
T=\frac{E_{1}}{2 \cdot p_{\max }} \geq \frac{D_{\mathrm{TSP}}}{V}+\frac{N \cdot E_{1}}{U-p_{\max }}
$$

Then, we can rewrite the inequality in (23) to the following quadratic inequality which is defined as the function $f\left(p_{\max }\right)$,

$$
f\left(p_{\max }\right)=A \cdot p_{\max }^{2}-(A U+B) \cdot p_{\max }+C \geq 0
$$

where $A=2 D_{\mathrm{TSP}} / V, B=(2 N+1) E_{1}$ and $C=E_{1} \cdot U$. Define $x_{1}$ and $x_{2}\left(x_{1} \leq x_{2}\right)$ as the two roots of the

Table 6 The result of the classified set $S_{k}(k=1,2, \ldots, r)$ for the 100-node network

\begin{tabular}{ll}
\hline Set Index & Node Index \\
\hline$S_{1}$ & $6,33,45,84,87$ \\
$S_{2}$ & $7,25,47,71,86$ \\
$S_{3}$ & $4,15,20,23,24,36,65,74,92$ \\
$S_{4}$ & $3,30,32,43,52,53,55,73,90,93$ \\
$S_{5}$ & $5,8,10,13,26,28,29,35,38,42,59,64,78,85,95,100$ \\
$S_{6}$ & $12,17,51,67,69,70$ \\
$S_{7}$ & $1,2,21,34,58,60,62,63,75,77,82,83,88,94,97,98$ \\
$S_{8}$ & $18,27,39,41,46,49,50,61,68,79,80,81,89,96$ \\
$S_{9}$ & $14,16,22,40,48,54,72,76,99$ \\
$S_{10}$ & $9,11,37,44,57,91$ \\
$S_{11}$ & $19,31,56,66$ \\
\hline
\end{tabular}

equation $f\left(p_{\max }\right)=0$. Then, the inequality in (23) can be established if $p_{i}$ satisfies the following relationship,

$$
p_{i} \leq p_{\max } \leq x_{1}=\frac{A U+B-\sqrt{(A U+B)^{2}-4 A C}}{2 A}
$$

Then, we can obtain the Theorem 1 by bringing $B$ and $C$ into (25). This completes the proof.

\section{Conclusion}

In this paper, we studied the operation of a sensor network based on WET, in a scenario where a MCV was employed to charge the sensor nodes wirelessly inside the network. We analyzed the energy consumption of the entire system and pointed out that the traveling distance of MCV is the main factor influencing total energy consumption. Based on energy consumption analysis and periodic strategy, we proposed a scheme ERSVC to decrease total energy consumption while maintaining the network operational forever with low complexity. Compared to previous schemes where the MCV visits and charges all nodes in each cycle, the MCV only needs to visit a portion of nodes in ERSVC by taking into the account the difference of energy consumption rate at each node. Therefore, the traveling distance of $\mathrm{MCV}$ was significantly decreased, and the total energy consumption could be saved. In ERSVC, we firstly formulated a practical optimization problem with flow rate to obtain the energy consumption rate, then designed the system for combining the charging period of each node, the visiting set, and traveling path during each cycle.

Table 7 Comparison of performance for proposed ERSVC and TSCC in the 100-node network

\begin{tabular}{llll}
\hline Parameter & Proposed ERSVC & Traditional TSCC & Scheme in [9] \\
\hline$\overline{L_{\text {total }}(m)}$ & 1809 & 7405 & 5746 \\
$P_{\text {total }}$ & 12.47 & 25.3 & 21.6 \\
$\eta_{\text {vac }}$ & $86.21 \%$ & $85.95 \%$ & $85.77 \%$ \\
\hline
\end{tabular}




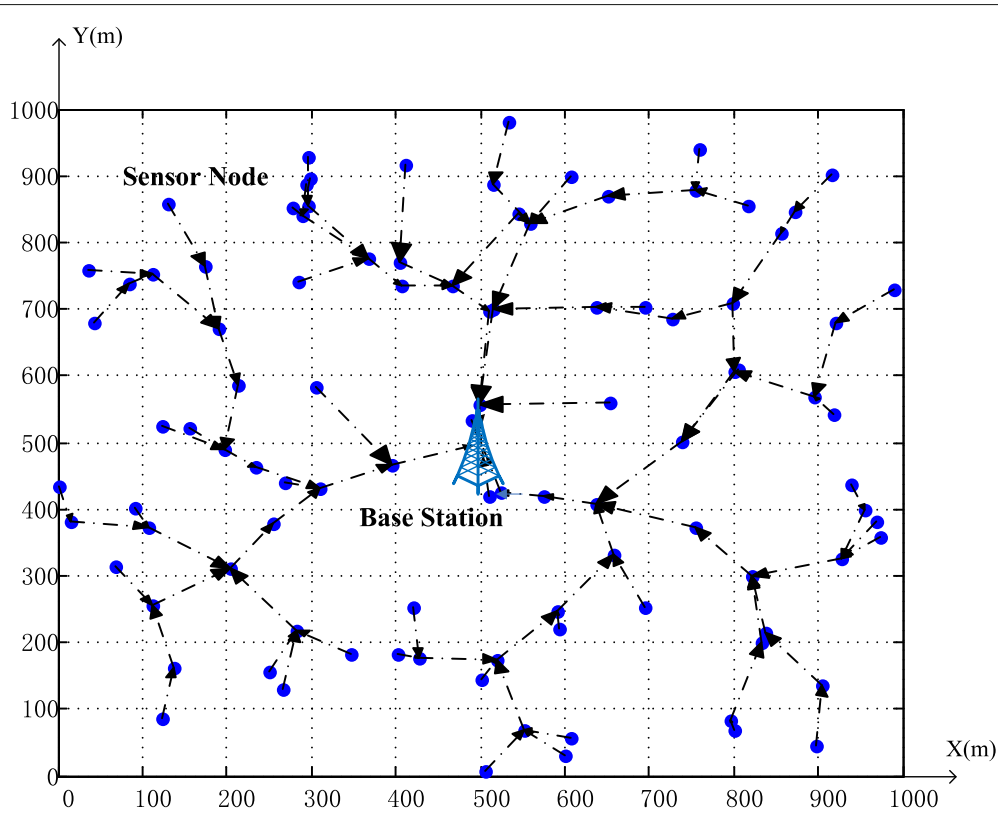

Fig. 7 The energy behavior of node $i$ during the $n$th and $(n+1)$ th charging period. $T_{i}^{\prime}$ is defined as the visiting and charging interval of $i$, which is the time interval between two adjacent arrivals at $i$ by MCV. In ERSVC, the MCV only visits and charges a portion of nodes in each cycle, and the visiting set in each cycle is different. Therefore, the moment when MCV arrives at $i$ is different every time, and the visiting interval $T_{i}^{\prime}$ is variable. In Fig. 7, $a_{i}$ is the moment when MCV arrives at node $i$ in the $(n+1)$ th charging period. After the time of $t_{i}$, the MCV charges the battery of node $i$ to $E_{\max }$. Apparently, the slope is $\left(U-p_{i}\right)$ when the MCV is charging node $i$ and the slope is $p_{i}$ at other times

Subsequently, we gave the proof that ERSVC can guarantee the network operational permanently. Simulations were carried out, showing that ERSVC can significantly decrease the total energy consumption with no performance loss in terms of the vacation time ratio.

The limitation of this work is that the flow rate is assumed to be invariant with time in order to simplify the optimization. However, it is time-varying in many real applications. In addition, we multiply the parameter $T$ by coefficient $1 / 2$ due to the variability of $T_{i}{ }^{\prime}$ which will degrade the energy saving performance. This can be improved by using the heuristic approach, such as genetic algorithm and ant colony algorithm. We will investigate these problems in our future research.

\section{Acknowledgements}

This work is supported by the National Natural Science Foundation of Guangdong Province in China with No. 2016A030310027.

\section{Authors' contributions}

Qian Zhang designed the system model and the implementation of the proposed scheme. Rong Cheng collected the relative literatures and performed some experiments to obtain the numerical results. Zhihua Zheng was a major contributor in writing and revising the manuscript. All authors read and approved the final manuscript.

\section{Availability of data and materials}

Data sharing is not applicable to this article as no datasets were generated or analyzed during the current study.

\section{Competing interests}

The authors declare that they have no competing interests.
Received: 17 November 2019 Accepted: 6 March 2020

Published online: 03 April 2020

\section{References}

1. Y.T. Hou, Y. Shi, H. D. Sherali, Rate allocation and network lifetime problems for wireless sensor networks. IEEE/ACM Trans. Netw. 16(2), 321-334 (2008)

2. E. M. Malika, B. C. Haithem, A. Rabah, Prolonging the lifetime of large-scale wireless sensor networks using distributed cooperative transmissions. IET Wirel. Sens. Syst. 8(5), 229-236 (2018)

3. J. Zhu, H. Cao, C. Song, Z. Xu, Parameter estimation via unlabeled sensing using distributed sensors. IEEE Commun. Lett. 21(10), 2130-2133 (2017)

4. T. Ruan, C. Zheng, M. Zhu, Energy-aware approaches for energy harvesting powered wireless sensor nodes. IEEE Sens. J. PP(99), 1-1 (2017)

5. K. André, K. Aristeidis, M. Robert, J. D. Joannopoulos, et al., Wireless power transfer via strongly coupled magnetic resonances. Science. 317(5834), 83-86 (2007)

6. L. Xie, S. Yi, Y. T. Hou, H. D. Sherali, Making sensor networks immortal: an energy-renewal approach with wireless power transfer. IEEE/ACM Trans. Netw. 20(6), 1748-1761 (2012)

7. L. Xie, Y. Shi, Y. T. Hou, W. Lou, et al., in IEEE Commu. Society Conf. on Sensor, Mesh and Ad Hoc Commun. and Networks. On renewable sensor networks with wireless energy transfer: the multi-node case, (2013). https://doi.org/ 10.1109/secon.2012.6275766

8. L. Xie, S. Yi, Y. T. Hou, W. Lou, et al., A mobile platform for wireless charging and data collection in sensor networks. IEEE J. Sel. Areas Commun. 33(8), 1521-1533 (2015)

9. L. Kaiyang, P. Jun, H. Liang, P. Jianping, et al., An active mobile charging and data collection scheme for clustered sensor networks. IEEE Trans. Veh. Technol. 68(5), 5100-5113 (2019)

10. S. He, J. Chen, F. Jiang, D. K. Y. Yau, et al., Energy provisioning in wireless rechargeable sensor networks. IEEE Trans. Mob.Comput. 12(10), 1931-1942 (2013)

11. B. Jin, J. Zhu, Q. Wu, Y. Zhang, et al., One-bit LFMCW radar: spectrum analysis and target detection. To appear in IEEE Transactions on Aerospace and Electronic Systems 
12. W. M. Ng, C. Zhang, D. Lin, S. Y. R. Hui, Two- and three-dimensional omnidirectional wireless power transfer. IEEE Trans. Power Electro. 29(9), 4470-4474 (2014)

13. M. Niendorf, P. T. Kabamba, A. R. Girard, Stability of solutions to classes of traveling salesman problems. IEEE Trans. Cybern. 46(4), 973-985 (2016)

14. S. Shafiee, M. Fotuhi-Firuzabad, M. Rastegar, Investigating the impacts of plug-in hybrid electric vehicles on power distribution systems. IEEE Trans. Smart Grid. 4(3), 1351-1360 (2013)

15. Concorde TSP Solver. http://www.math.uwaterloo.ca/tsp/concorde/ index.html. Accessed Mar 2015

\section{Publisher's Note}

Springer Nature remains neutral with regard to jurisdictional claims in published maps and institutional affiliations.

\section{Submit your manuscript to a SpringerOpen ${ }^{\odot}$ journal and benefit from:}

- Convenient online submission

- Rigorous peer review

- Open access: articles freely available online

- High visibility within the field

- Retaining the copyright to your article

Submit your next manuscript at $\gg$ springeropen.com 